\title{
Improved ASCAT wind retrieval using NWP
}

\section{ocean calibration}

\author{
J. Verspeek ${ }^{(1)}$, A. Stoffelen ${ }^{(1)}$, A. Verhoef ${ }^{(1)}$, M. Portabella ${ }^{(2)}$
}

Abstract - The Advanced Scatterometer (ASCAT) Wind Data Processor (AWDP) currently uses the so called CMOD5n Geophysical Model Function (GMF), which was originally derived for the ERS scatterometers. In order to deliver a highquality ASCAT wind product, the operational AWDP uses backscatter measurement corrections that are estimated visually (VOC) for each wind vector cell (WVC). We propose an alternative and previously established method for estimating correction tables based on NWP ocean calibration residuals (NOC). It embodies a smooth incidence-angle dependent part that could serve as an appropriate ASCAT GMF correction, and a radar-beam dependent residual. The incidence-angle-dependent part of these correction tables is due to differences in calibration procedure of the ERS and ASCAT scatterometers. For the high ASCAT incidence angles for which the GMF has not been assessed by ERS data the modification is quite large, almost $1 \mathrm{~dB}$. The incidence angle dependent part is derived by fitting the ocean calibration residuals of all beams obtained over one year of data. It is subsequently used to adapt the GMF (yielding CMOD5na). The remaining radar-beam dependent residual (NOCa) shows a wiggle pattern as function of incidence angle that is very persistent over time, apart from a seasonally varying offset. Both the effects of the GMF modification and the beamdependent residual on the wind retrieval quality are investigated in this paper. Overall the performance of NOC is better than that obtained with the previously used VOC calibration method and the wind statistics show a much better symmetry of the left and right swath for NOC. The beam-dependent corrections improve the quality of the retrieved winds. NOC may thus be used for the intercalibration of the ERS and ASCAT scatterometers.

\footnotetext{
(1) Royal Netherlands Meteorological Institute (KNMI), Wilhelminalaan 10,3732 GK De Bilt, The Netherlands (2) Unidad de Tecnología Marina (UTM - CSIC),
} 
Index Terms - Calibration, Radar scattering, Wind, Scatterometer.

\title{
I. INTRODUCTION
}

\begin{abstract}
$\mathbf{T}$ HE METOP-A satellite was launched on October 19, 2006 and carries ASCAT. This instrument is a real-aperture C-band
\end{abstract} vertically polarized radar with three fan beam antennas pointing to the left-hand side of the sub satellite track and three fan beam antennas pointing to the right-hand side [1]. A measurement space is defined for each Wind Vector Cell (WVC) as the threedimensional or $3 \mathrm{D}\left(z_{\text {fore }}, z_{\text {aft }}, z_{\text {mid }}\right)$ space where $z=\sigma_{0}^{0.625}$, the transformed measured backscatter value of respectively the fore, aft and mid beam [2]. The two-dimensional (2D) GMF manifold is a conical surface and the (fore, aft, mid) measurement triplets generally lie in the proximity of this surface.

Fig. 1 shows a visualization of the measurement space. The backscatter triplets are shown as black points. Differences between the cloud of triplets and the cone in each direction of the measurement space are, to a first approximation, caused by biases between the ASCAT data and the ERS-based GMF. The blue double folded conical surface represents CMOD5n, the GMF that is currently being used in the operational wind processing. CMOD5n is a modification of CMOD5 that was used for ASCAT and ERS wind processing in the past. Although the shape of the surface is the same for both CMOD5 and CMOD5n, the latter relates backscatter to equivalent neutral winds at 10-m rather than to real winds and is a better representation of the geophysical quantity that is measured by the scatterometer (not depending on atmospheric stability). The GMF is introduced further in section II.

\footnotetext{
Pg. Marítim Barceloneta 37-49, 08003 Barcelona, Spain
} 


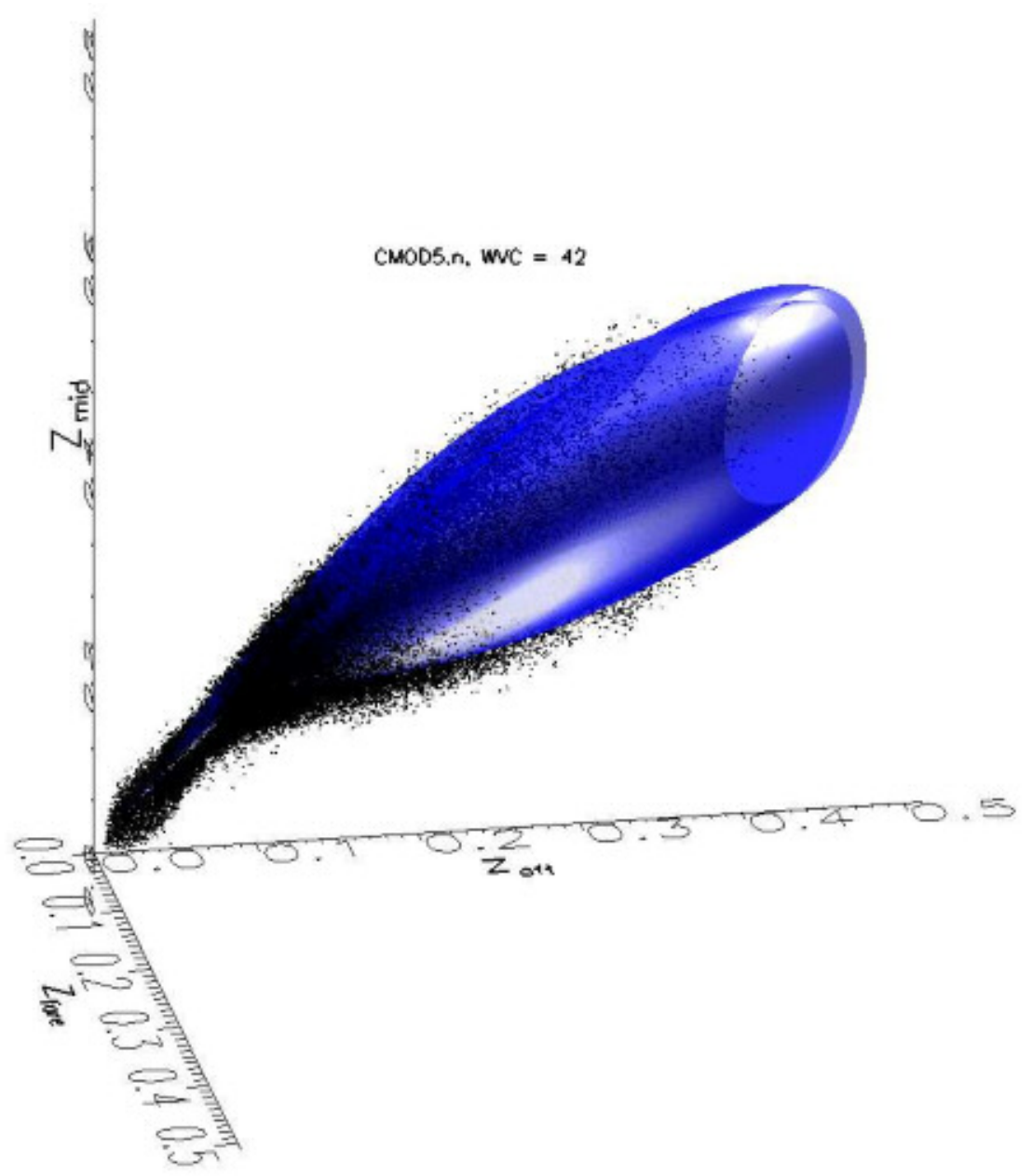

Figure 1 - Visualization of CMOD5.n and the ASCAT triplets in 3D measurement space for the innermost WVC of the right swath (WVC 42, $12.5 \mathrm{~km} \mathrm{WVC} \mathrm{spacing)} \mathrm{for} \mathrm{wind} \mathrm{speeds} \mathrm{up} \mathrm{to} 30 \mathrm{~ms}-1$.

The AWDP uses corrections in order to achieve a high quality wind product. The currently used corrections are based on a Visual correction method for Ocean Calibration (VOC) [3]. In this method the Geophysical Model Function (GMF) is evaluated in the measurement space for its consistency with the distribution of measurement points. The VOC method scales each of the three axes of the measurement space, i.e., the fore, aft and mid beam $\sigma_{0}$, such that the distribution of measurement triplets is shifted towards the conical GMF surface as defined by CMOD5n. As a second correction, the axes are scaled simultaneously to compensate for a wind speed bias with respect to collocated ECMWF model winds. Thus instrumental or calibration errors as well as GMF defects can be accounted for.

Another method for Ocean Calibration (OC) resides in direct comparison of measured $\sigma_{0}$ data with simulated values from Numerical Weather Prediction (NWP) model winds using the GMF (see [2], [4]). This NWP-based OC (NOC) method, that was 
also used for ERS scatterometers in the past, and for assimilation of ASCAT winds in the ECMWF model (see [6], section 4), is explained in section II. Section III describes the derivation of the NOC backscatter correction factors. The NOC corrections are furthermore split into an incidence angle dependent part, which is included in the GMF, and a remaining antenna-dependent part (the NOCa corrections). In section IV, the impact of both VOC and NOCa antenna-dependent corrections on several windinversion related parameters is assessed. Metrics based on the statistical distribution of several parameters will be discussed and evaluated in section V. Section VI gives the summary and conclusions.

\section{NWP OCEAN CALIBRATION}

The NOC method is based on the analysis of a large measurement dataset to estimate Fourier coefficients that can be directly compared to those in the CMOD5.n GMF which may be written as ([5], [6], [7]):

$$
\left.\sigma_{0}(v, \theta, \phi)=B_{0}(v, \theta)\left[1+B_{1}(v, \theta) \cos \phi+B_{2}(v, \theta) \cos (2 \phi)\right]^{1.6} \text { (eq. } 1\right)
$$

where $v$ is the wind speed, $\phi$ the wind azimuth direction relative to the radar beam look, and $\theta$ the radar incidence angle. The $\mathrm{B}$ coefficients are closely related to Fourier coefficients in $\mathrm{z}$ space $\left(z=\sigma_{0}^{0.625}\right)$ such that Eq. 1 can be re-written as:

$$
\left.z(v, \theta, \phi)=\frac{1}{2} a_{0}(v, \theta)+a_{1}(v, \theta) \cos \phi+a_{2}(v, \theta) \cos (2 \phi) \quad \text { (eq. } 2\right)
$$

When the wind direction distribution is sampled uniformly for all wind speeds, then the mean $<(1 / 2) a_{0}>$ should be identical to the mean $\left\langle z>\right.$. This means that uncertainties in $a_{1}$ and $a_{2}$ do not contribute to the error in the estimated mean $z$.

In this paper only the ASCAT high resolution mode is used $(12.5 \mathrm{~km}$ WVC spacing compared to the $25 \mathrm{~km}$ WVC spacing for the nominal resolution). To arrange a uniform wind direction distribution, we split the collocated European Centre for Medium-range Weather Forecasts (ECMWF) wind data into wind speed bins of size $1.0 \mathrm{~ms}^{-1}$ and azimuth angle bins of size $12^{0}$. Our operational product uses ECMWF winds (3-hourly 3-18 hour forecast winds) at spectral truncation T319 (corresponding to a grid with 160 points between pole and equator) interpolated to ASCAT wind locations and time. For the ocean calibration these ECMWF forecast winds are used. Data from the global oceans between latitudes $-55^{0}$ and $+65^{0}$ are used. The latitudes are chosen such that 
1

2

3

4

5

6

7

8

9

regions which possibly contain sea ice are conservatively excluded. The collocated ECMWF equivalent neutral 10-meter winds are converted to simulated $\mathrm{z}$ values using the CMOD5.n GMF (Eq. 2). The differences of measured and simulated $<\mathrm{z}>$ are averaged over all wind azimuth bins weighted in accordance with a uniform wind azimuth distribution. Next they are averaged over all wind speed bins weighted in accordance with the wind speed distribution. Thus, the NOC method needs only a few days of collocated ASCAT data and ECMWF winds to produce a reasonable estimate of difference in backscatter residuals, i.e., the difference between the two values of $<\mathrm{z}>$ as a function of incidence angle for each antenna. When these residuals are stable over time they may be used as correction factors for errors in the instrument, for monitoring instrument health or for GMF development. Stoffelen [8] notes that uncertainties in ECMWF wind speed and direction may cause small systematic biases in the residuals produced by the ocean calibration. Therefore, it is important to verify the effect of NOC in the scatterometer wind retrieval (see section IV) and other applications.

A time series of the ocean calibration is performed over the period of one year, from September 2008 to September 2009 for the ASCAT scatterometer in high-resolution mode (12.5 km WVC spacing). The one-year period is taken to average out the seasonal variations in the wind distribution that have an effect on the NOC residual. Successive periods of day 1-14 and day 15-last day of the month are taken as input for an ocean calibration run. The ASCAT backscatter data (level 1B product) is provided by EUMETSAT. Since the beginning of ASCAT operations, the calibration of level 1B data has been adjusted several times as additional transponder data was collected and calibration algorithms were refined. Corrections that account for differences in level 1B versions are applied to the backscatter data. These corrections have been able to transform the ASCAT backscatter measurements from each level 1B calibration cycle to the next cycle within a few hundredths of a dB (see [9] for details). Thus the results are made independent of the level 1B software version that is used. Level 1B software version 7.02 based on the 3transponder calibrated data [10], [11] is taken as the reference by NOC.

Fig. 2 shows a typical example the ocean calibration residuals from the right-fore antenna as a function of incidence angle. Each line corresponds to a time period of half a month. The figure shows a good stability over time. The vertical fluctuations between lines are in the order of $\sim 0.1 \mathrm{~dB}$, corresponding approximately to $\sim 0.1 \mathrm{~m} / \mathrm{s}$ in the wind speed domain. These fluctuations are apparent for the whole incidence angle range. Seasonal variations in NWP wind distribution over the year may introduce a small varying bias in OC residuals and are the main cause of these fluctuations in the vertical axis direction [8]. The pattern within one line as a function of incidence angle shows distinct peaks and troughs. These are difficult to explain from the NWP comparison 
procedure [2], [8] since the GMF terms are rather smooth as a function of incidence angle and subsequent WVCs see almost identical NWP wind distributions. Moreover, variations in wind distribution are not expected to be fixed with respect to WVC from one month to the next. The small wiggles are presumably ASCAT instrument calibration artifacts. Also for the other antennas a peculiar incidence-angle-dependent pattern exists that is stable over time, with a very similar vertical shift over time (see also Anderson et al [12]).

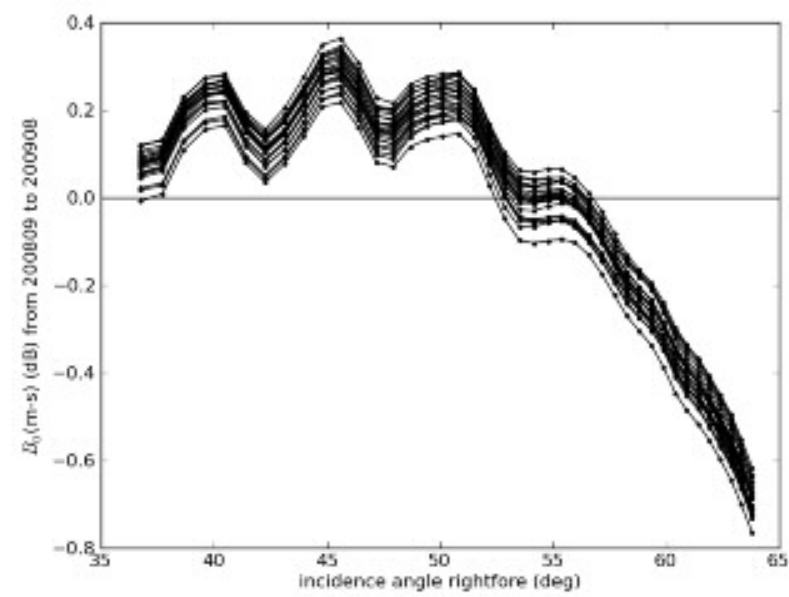

Figure 2 - Stability over time of the right-fore antenna NWP ocean calibration residual as a function of incidence angle (values converted to $\mathrm{dB}$ ) for the $12.5-\mathrm{km}$ product.

\section{DERIVATION OF THE NOCa CORRECTION FACTORS}

From a physical point of view the backscatter is a smooth and slowly varying function of incidence angle and no wiggles with period $\sim 5$ degree as appear in Fig. 2 are to be expected. Similar wiggles also appear in independent rain forest study [12] and in averaged backscatter from the scatterometer alone without using the collocated ECMWF winds. The wiggles could be caused by level 1B calibration inaccuracies, but could also be introduced in the calculation of the level $1 \mathrm{~B} \sigma_{0}$ value out of the level 0 radar data product, e.g. by inaccuracies in the antenna pointing or footprint area calculation. The NOC corrections are split into a main smooth incidence-angle dependent part $B_{0}{ }^{\text {corr }}(\theta)$ that is attributed to the wind GMF and a relatively small remaining beamdependent part (NOCa) that is attributed to instrument or level $1 \mathrm{~B}$ calibration deficiencies. The latter corrections could be used as correction factors for other geophysical products as well, e.g. soil moisture [13] and sea ice [14]. 
Fig. 3(a) shows the average of the NOC residuals over a time series of one year that define the NOC correction factors. The thick line is a fit function $B_{0}{ }^{\text {corr }}(\theta)$ through the NOC correction factors for all six antennas. It is a third order polynomial in the incidence angle $\theta$ over the total range from $27.5^{\circ}$ to $63.6^{\circ}$. Especially for the high incidence angles for which the GMF has not been assessed by ERS data the fit function has a quite large value, in the order of $1 \mathrm{~dB}$ lower than for the mid range incidence angles. The correction function $B_{0}{ }^{\text {corr }}(\theta)$ is incorporated into the ASCAT GMF to yield CMOD5na:

$$
\begin{aligned}
& \text { CMOD5na }=\text { CMOD5n }+B_{0}^{\text {corr }}(\theta) \\
& B_{0}^{\text {corr }}(\theta)=a_{0}+a_{1} \theta+a_{2} \theta^{2}+a_{3} \theta^{3}
\end{aligned}
$$

with polynomial coefficients $a_{0}=5.7236425879, a_{1}=-0.4226930560, a_{2}=0.0105605079, a_{3}=-0.0000864832$.

Fig. 3(b) shows the zoomed-in differences between NOC factors and the fit function. Clearly, remaining instrument calibration wiggles can be depicted in the range of $-0.15 \mathrm{~dB}$ to $+0.15 \mathrm{~dB}$. These remaining factors are antenna dependent and therefore cannot be incorporated in the GMF. They will be referred to as NOCa corrections.

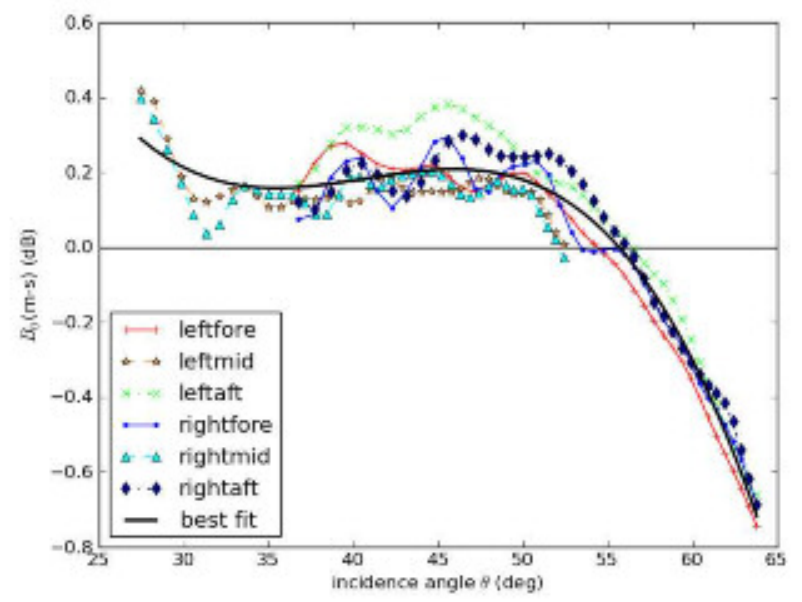

a) 


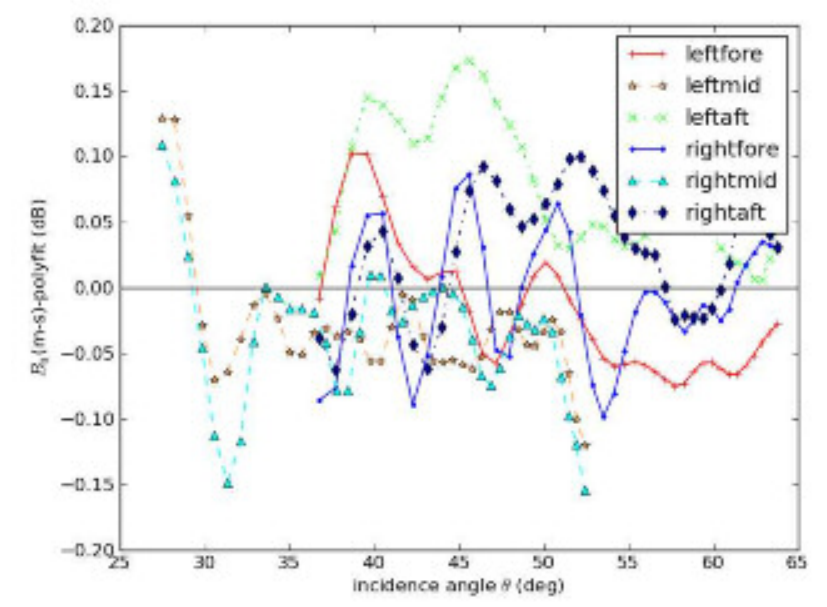

b)

\section{Figure 3 -}

a) Average of the ocean calibration residuals over one year (NOC correction factors). A polynomial fit through the NOC calibration corrections is also shown.

b) NOCa corrections. Difference between NOC corrections and polynomial fit. i.e., residual corrections for CMOD5na.

All the computed 12.5 -km product residual values are converted from linear space to dBs.

In this paper only the high resolution mode is used (12.5 km WVC spacing) [15]. The NOCa residuals for high resolution reveal more detail than the residuals for nominal resolution ( $25 \mathrm{~km} \mathrm{WVC} \mathrm{spacing).} \mathrm{Using} \mathrm{nominal} \mathrm{mode} \mathrm{corrections} \mathrm{that} \mathrm{are} \mathrm{derived}$ from the high resolution mode corrections would lead to insufficient accuracy at the swath edges. NOCa corrections are also calculated for the nominal resolution mode, yielding separate correction tables for high and nominal resolution.

\section{COMPARISON OF THE WIND STATISTICS WITH NOCa AND VOC CORRECTIONS}

In this section we compare ASCAT wind statistics from reprocessed wind products (first week of February 2009) by AWDP using CMOD5na and NOCa corrections with the operational values where CMOD5.n with VOC corrections were applied.

Fig. 4(a) shows the wind speed bias as a function of WVC from CMOD5na+NOCa, CMOD5na and CMOD5n+VOC corrected data with respect to the NWP winds from ECMWF. The VOC corrected bias is generally further away from zero. Moreover, it 
1

2

3

4

5

6

7

8

9

shows an asymmetric pattern for the left and right swath. The NOCa corrected bias is generally closer to zero and it shows a symmetrical pattern for the left and right swath as expected. The impact of the NOCa corrections can be seen by comparing CMOD5na+NOCa with CMOD5na. The overall pattern is the same, but the NOCa corrections have clearly removed the irregularities that can be seen for the CMOD5na case. Fig. 4(b) shows the $u$ and $v$ wind component Standard Deviation (SD) of the difference between ASCAT winds and collocated NWP winds as a function of WVC. The patterns are comparable for the NOCa and the VOC correction case, but are systematically lower for the NOCa case, denoting improved wind retrieval for NOCa. Table 1 summarises the wind statistics in terms of bias and standard deviation for the two cases with respect to NWP winds. The NOCa case has better statistics than the operational case (VOC). Biases are comparable, but SD values for wind speed $V$, wind direction $\phi$ and wind components $u$ and $v$ are all slightly lower for NOCa.

Validation of ASCAT winds with an independent source is performed via triple collocation studies in which ASCAT winds are compared with e.g. ECMWF winds and buoy winds. In [16], the ASCAT operational wind product (VOC) from one year of data is used in such a triple collocation study. In the context of this paper, triple collocation studies have been performed for both NOCa and VOC ASCAT winds from February 2009 with ECMWF and buoy winds. The resulting wind statistics from NOCa and VOC data are alike, with almost identical SDs for the $u$ and $v$ wind components (not shown). Thus the results from [16] for VOC ASCAT winds are applicable for NOCa as well.

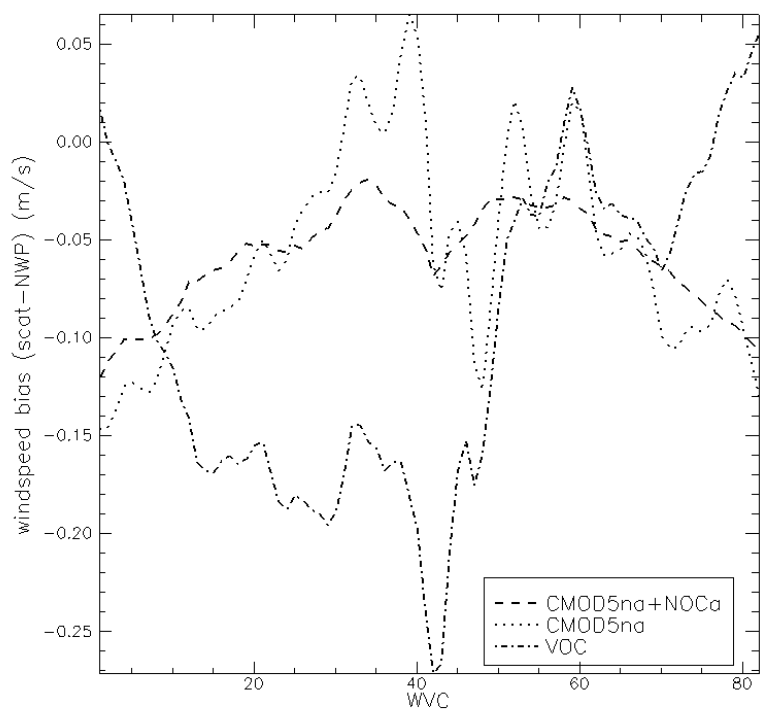

a) 


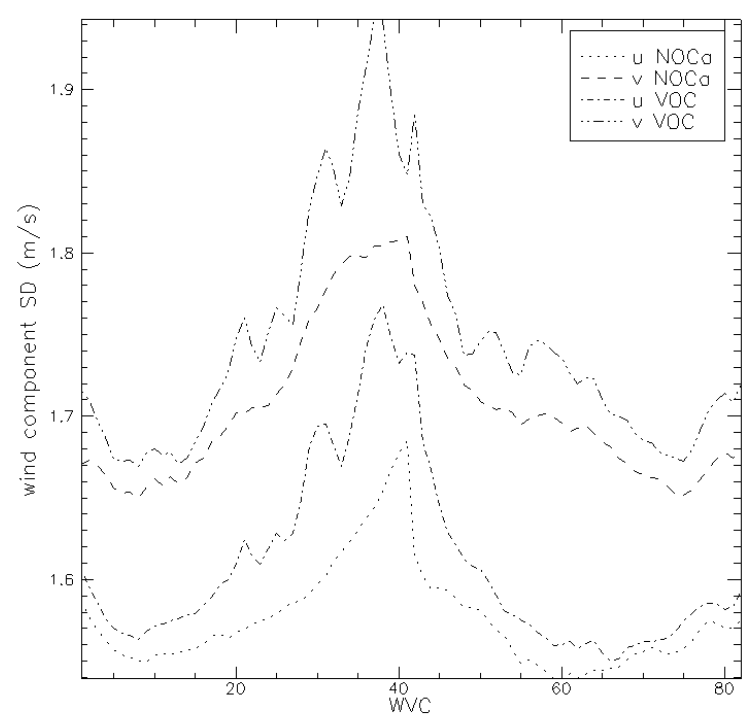

b)

Figure 4 -

a) Wind speed bias $\left(\mathrm{V}_{\text {scat }}-\mathrm{V}_{\text {nwp }}\right)$ as a function of WVC. ASCAT data from the first week of February 2009 was processed using CMOD5na in combination with NOCa, and CMOD5n in combination with VOC corrections.

b) Standard deviation of the wind component difference $\operatorname{SD}\left(u_{\text {scat }}-u_{\text {nwp }}\right)$ and $\operatorname{SD}\left(v_{\text {scat }}-v_{\text {nwp }}\right)$ as a function of WVC.

Table 1 - Wind statistics for NOCa and VOC correction (ASCAT data from the first week of February 2009)

\begin{tabular}{|l|l|l|l|l|}
\hline & Bias NOCa & Bias VOC & SD NOCa & SD VOC \\
\hline V & -0.06 & -0.10 & 1.31 & 1.32 \\
\hline$\phi$ & 0.49 & 0.74 & 16.38 & 16.98 \\
\hline $\mathrm{u}$ & -0.11 & -0.09 & 1.58 & 1.61 \\
\hline $\mathrm{v}$ & -0.05 & -0.05 & 1.71 & 1.75 \\
\hline
\end{tabular}

The Maximum Likelihood Estimate (MLE) is the distance from a measurement triplet to the point on the wind cone in 3D measurement space that corresponds to the retrieved wind (see fig. 1). It is a measure of how well the measurements and GMF fit to each other. Due to instrument and geophysical characteristics of the scatterometer, the expectation value $<$ MLE $>$ is varying as a function of incidence angle or WVC. For the ease of monitoring and quality control (QC) the MLE is normalised using a WVC dependent factor, yielding an expectation value of $\langle\mathrm{MLE}\rangle=1$ for each WVC. In cases where a measurement triplet does not 
represent the wind well, e.g. due to ice contamination, the MLE will have a large value. When the MLE surpasses a certain threshold value, it is flagged by quality control (GMF_distance flag is set) [17].

NOCa and VOC use different MLE normalization and QC threshold tables and therefore a direct comparison of the MLE average/SD values is not fair. In order to make a fair comparison between NOCa and VOC, the ASCAT wind product is reprocessed using no MLE normalisation factors and the same QC threshold values in both cases. The resulting (non-normalised) MLE average/SD value is shown in fig. 5. Without normalisation the MLE distribution shows variations as a function of WVC that can be related to GMF errors, backscatter calibration errors, and the exact 3D shape of the GMF cone in 3D measurement space. In the NOCa corrected case any WVC dependency caused by small interbeam biases is already corrected out by the NOCa corrections itself. As such, the MLE is a smooth and symmetrical function. On the other hand, the VOC case shows asymmetry and irregularities. Also, from fig. 5 it can be concluded that both the mean MLE value and its SD are smaller everywhere for NOCa, which shows that the NOCa corrected data better fit the GMF cone.

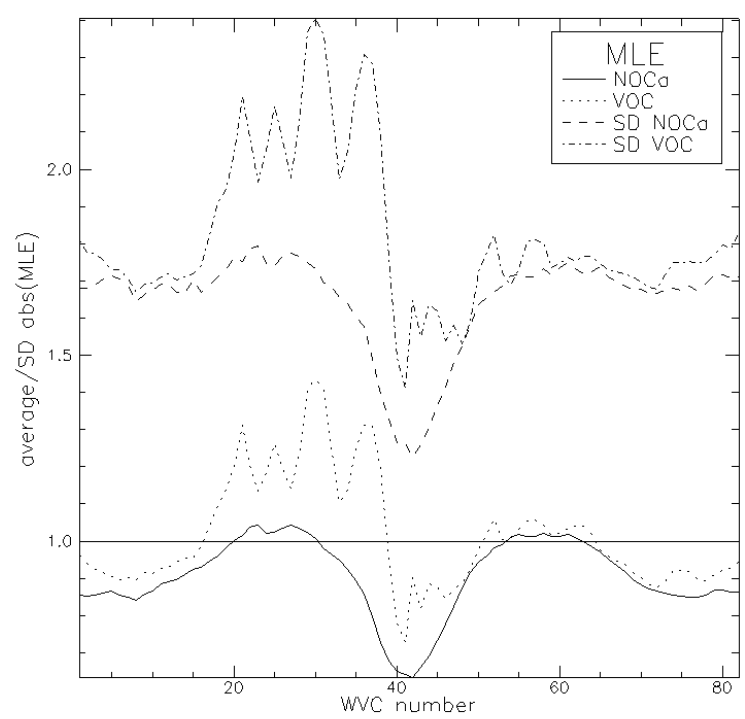

Figure 5 - Average/SD of the absolute value of the MLE per WVC. NOCa and VOC case are shown. ASCAT data from the first week of February 2009 was processed without using MLE normalisation factors, and with the same QC thresholds applied for both cases. 
During wind retrieval (level 2) processing QC flags may be set to indicate special or anomalous conditions [18], [19]. The GMF_distance flag is set when the measured triplet has an anomalously large distance to the GMF cone, while the var_qc flag is set during 2DVAR ambiguity removal when a wind vector is spatially inconsistent with its neighbours [20]. The KNMI QC flag is an overall level 2 QC flag incorporating several other flags [21].

The occurrence of some important level 2 quality flags and their WVC dependency is shown in fig. 6 for the NOCa case. Backscatter calibration causes (small) changes to the cone location in measurement space and thus requires new MLE normalisation. MLE normalisation and QC threshold tables were recomputed using NOC corrections (see annex of [9]). The difference with the VOC case (not shown) is small but again favourable for NOCa with a smoother curve for the KNMI QC flag and GMF_distance flag and less points rejected by the 2DVAR spatial inconsistency flag (var_qc).

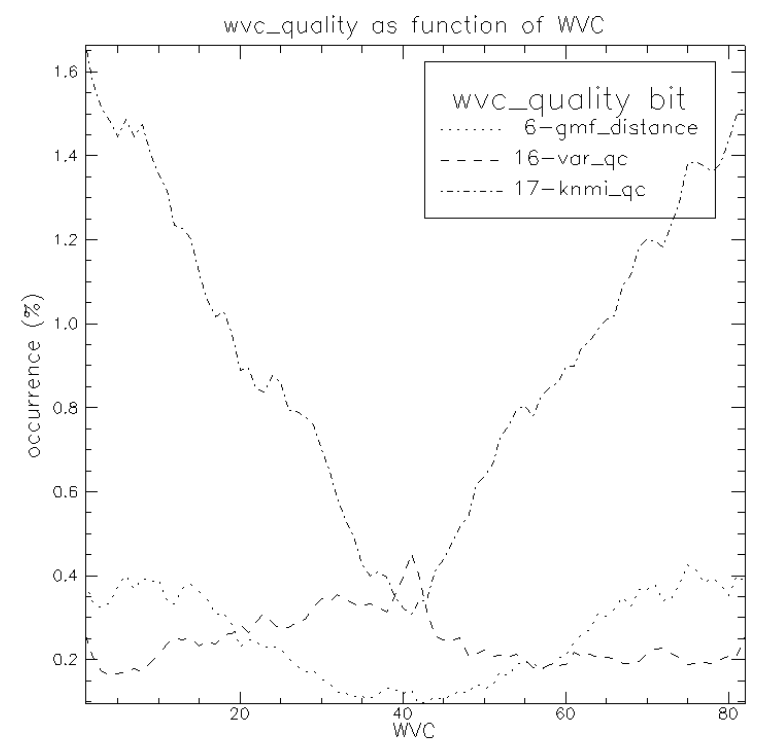

Figure 6 - Relative occurrence of some KNMI quality flags as a function of WVC. Data corrected with NOCa.

V. SUMMARY AND CONCLUSIONS

In many aspects the NOCa provides results that are comparable with, or better than the VOC method: 
- The two-weekly OC residuals for data processed with CMOD5n and without applied corrections are varying within a small range of $\sim 0.1 \mathrm{~dB}$ from period to period over the full observed timeframe of one year (see fig.2). This shows the consistency in the approach for applying NOCa corrections;

- The AWDP wind speed bias against ECMWF is small, but becoming symmetric for the left and right swath when the NOCa corrections are applied (see fig. 4a);

- The AWDP minus ECMWF wind speed, direction and component SDs are reduced for NOCa as compared to the VOCcorrection processed winds (see fig. 4b);

- The MLE is reduced by up to $40 \%$ in certain WVCs when NOCa corrections are used as compared to the MLEs produced by AWDP with VOC corrections. Moreover, following expectation the MLE is becoming symmetric for the left and right swath when the MLE normalization factors are omitted (see fig. 5);

- The reduction in level 2 QC flag occurrences for NOCa-corrected AWDP compared to the VOC case is about $10 \%$ for the 2D-VAR spatial consistency check (see fig. 6);

The symmetrical and beam-independent part of the NOC correction is put into a new version of the GMF, CMOD5na. ASCAT data processed with CMOD5na form a good basis for further GMF improvements using MLE residual analyses as a function of incidence angle, wind speed and wind direction.

The VOC WVC- and beam-dependent correction method makes use of a visual correction, judged by eye, and an integral multiplication factor to correct the mean wind speed to ECMWF. With hindsight, the VOC method was not focused on the modal winds and too much tuned towards the more extreme winds occurring at the different WVCs.

The remaining small beam-dependent part (NOCa) can be ascribed to instrument or level1B calibration deficiencies. It compensates for any remaining beam-dependent error. Most notably it removes the WVC-dependency of wind and MLE statistics. The NOCa-corrected backscatter triplets indeed visually better fit the GMF cone in measurement space at the modal wind speeds (not shown). The NOCa corrections may be used as correction factors for other geophysical products as well, e.g., soil moisture and sea ice. Since ASCAT is thought to have a superior calibration to ERS, the incidence-angle dependent part can be used to correct CMOD5n. The corrected CMOD5n would in turn be useful to determine an improved objective ERS calibration, i.e., to perform intercalibration of the ERS and ASCAT instruments. 
Implementation of CMOD5na and NOCa corrections together with new MLE normalization factors is useful and leads to slightly better ASCAT winds, QC and MLE statistics where the asymmetry between left and right swath is diminished. The MLE normalisation results in appropriate QC thresholds and monitoring flag settings for the NOCa implementation.

Reprocessing the ASCAT wind product using the CMOD5na and NOCa corrections, as well as the newly derived MLE normalization and QC threshold tables, yields good-quality wind and MLE statistics, slighty better than with the VOC method. Moreover, the distributions are more symmetric for left and right swath.

Also in the experimental ultra-high resolution mode $(6.25 \mathrm{~km})$ CMOD5na and appropriate NOCa corrections are expected to result in better winds and improved statistics.

\section{ACKNOWLEDGMENT}

The authors wish to thank their colleagues from EUMETSAT and KNMI, Craig Anderson, Hans Bonekamp, Julia Figa and Jur Vogelzang, for their interest in this work, stimulating discussions, provision of data and helpful advice.

Footnote: This work is done in the framework of the Ocean and Sea Ice Satellite Application Facility (OSI SAF) and its visiting scientist scheme. The ASCAT winds produced at KNMI can be obtained free of charge from the OSI SAF web site. AWDP has been funded by EUMETSAT in the context of the NWP SAF and can be obtained free of charge from the NWP SAF web site (Helpdesk: scat@knmi.nl). 

1

2

\section{REFERENCES}

[1] . J. Figa-Saldaña et al, "The advanced scatterometer (ASCAT) on the meteorological operational (MetOp) platform: A follow on for European wind scatterometers", Can. J. Remote Sensing, Vol. 28, No. 3, pp. 404-412, 2002.

[2].A. Stoffelen and D. Anderson, "Scatterometer Data Interpretation: Measurement Space and Inversion”, J. Atm. and Ocean Techn., vol. 14, no. 6, pp. 1298$1313,1997$.

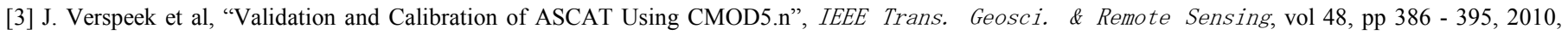
doi:10.1109/TGRS.2009.2027896.

[4] M. Freilich, H. Qi, and R. S. Dunbar, "Scatterometer beam balancing using open-ocean backscatter measurements," J. Atmos. Ocean. Technol., vol. 16, no. 2, pp. 283-297, Feb. 1999.

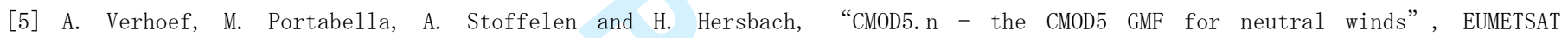
SAF/OSI/CDOP/KNMI/TEC/TN/165, 2008.

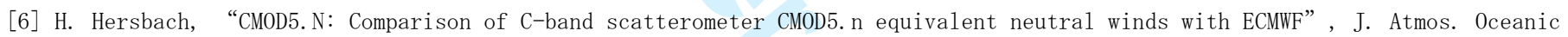
Technol., 27, 721-736. doi: 10. 1175/2009JTECH0698. 1

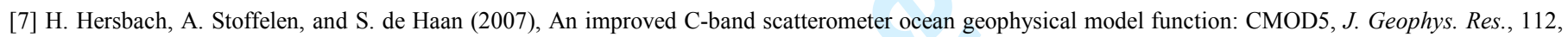
C03006, doi:10.1029/2006JC003743.

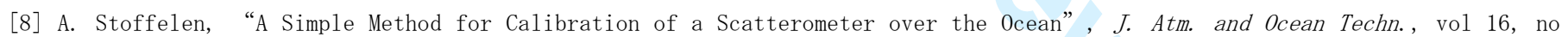
2, pp 275-282, 1999.

[9] J. Verspeek, A. Verhoef, A. Stoffelen, ASCAT NWP 0cean Calibration, 0SISAF report, KNMI, de Bi1t, 2011

[10] J. Wilson et al, "Radiometric calibration of the advanced wind scatterometer radar ASCAT carried on-board the METOP-A satellite", IEEE Trans. Geosci. \& Remote Sensing, vol 48 pp 3236 - 3255, 2010, doi: 10.1109/TGRS.2010.2045763.

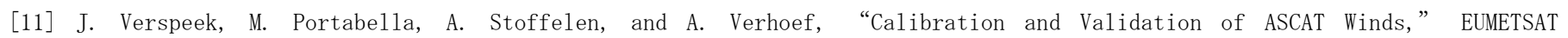
SAF/OSI/KNMI/TEC/TN/163, KNMI, de Bilt, 2008. 
[12] C. Anderson et al, "Validation of backscatter measurements from the Advanced Scatterometer on Metop-A", accepted for Journal of Ocean and Atmospheric Technology, 2011, doi: 10.1175/JTECH-D-11-00020.1

[13] V. Naeimi, K. Scipal, Z. Bartalis, S. Hasenauer and W. Wagner, "An improved soil moisture retrieval algorithm for ERS and METOP scatterometer observations", IEEE Transactions on Geoscience and Remote Sensing, Vol. 47, pp. 1999-2013., 2009, doi: 10.1109/TGRS.2008.2011617

[14] M. Belmonte Rivas and A. Stoffelen, "New Bayesian algorithm for sea ice detection with QuikSCAT", IEEE Transactions on Geoscience and Remote Sensing, Vol 49, no 6, pp. 1894-1901, 2011, doi:10.1109/TGRS.2010.2101608

[15] A. Verhoef and A. Stoffelen, "Validation of ASCAT 12.5-km winds", Ocean and Sea Ice SAF Technical Note SAF/OSI/CDOP/KNMI/TEC/RP/147, Version 1.2, February 2009. Available: www.knmi.nl/scatterometer/publications/pdf/ASCAT_validation_125.pdf

[16] J. Vogelzang, A. Stoffelen, A. Verhoef, and J. Figa-Saldana (2011), “On the quality of high-resolution scatterometer winds”, J. Geophys. Res., 116, C10033, doi:10.1029/2010JC006640

[17] M. Portabella, and A. Stoffelen, “Characterization of residual information for SeaWinds quality control," IEEE Trans. Geosci. Rem. Sens., 40 (12), doi:10. 1109/TGRS. 2002. 807750, pp. 2747-2759, 2002.

[18] M. Portabella and A. Stoffelen, "Scatterometer backscatter uncertainty due to wind variability", IEEE Transactions on Geoscience and Remote Sensing, Vol 44, no 11, pp. 3356-3362, 2006, doi:10.1109/TGRS.2006.877952.

[19] A. Stoffelen and M. Portabella, “On Bayesian Scatterometer Wind Inversion”, IEEE Transactions on Geoscience and Remote Sensing, Vol 44, no 6, pp. 1523-1533, 2006, doi:10.1109/TGRS.2005.862502

[20] J. Vogelzang et al, "Validation of two-dimensional variational ambiguity removal on Seawinds scatterometer data. ",J. Atmos. Oceanic Technol., in press, 2009

[21] A. Verhoef, A. and A. Stoffelen, ASCAT Wind Product User Manual version 1.9, SAF/0SI/CDOP/KNMI/TEC/MA/126, EUMETSAT, 2011

Jeroen Verspeek was born on July 2, 1962, in The Netherlands. He received his M.Sc. degree in physics from the Technical University of Eindhoven, Eindhoven, The Netherlands, in 1988.

He is currently with the Royal Netherlands Meteorological Institute (KNMI), De Bilt, The Netherlands, and is working on scatterometer data interpretation, inversion, calibration, validation and quality monitoring. 


\section{Page 23 of 24}

1

2

3

4

5

6

7

8

9

10

11

12

13

14

15

16

17

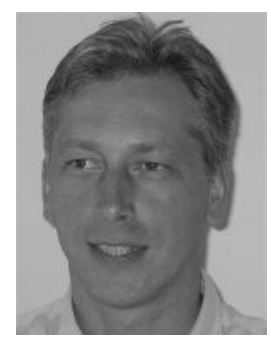

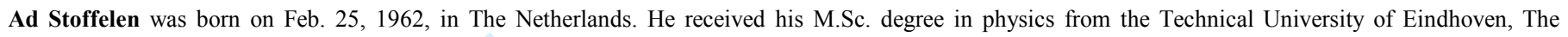
Netherlands, in 1987 and his Ph.D in Meteorology on scatterometry at the University of Utrecht, the Netherlands.

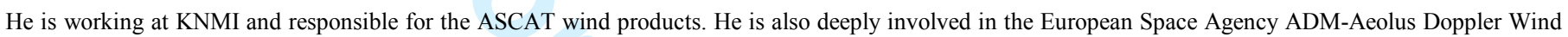
Lidar mission.

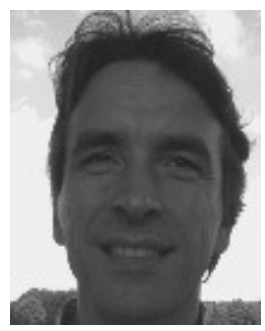

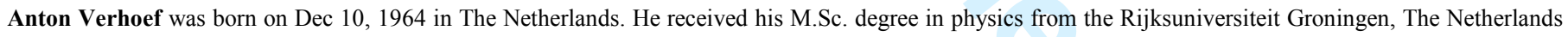
in 1989 and his Ph.D in Solid State Physics at the Rijksuniversiteit Groiningen in 1994.

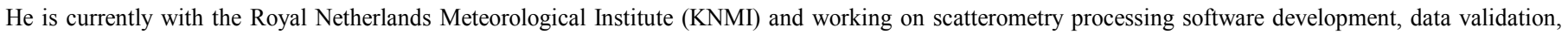
quality monitoring and user services.

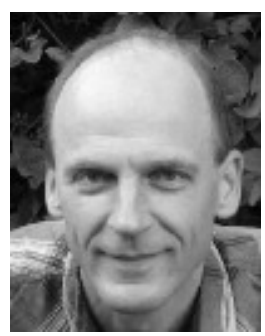


Marcos Portabella was born on October 14, 1970 in Spain. He received the B.Sc. degree in Physics in 1994 from the University of Barcelona, Spain; the M.Sc. in Remote Sensing in 1995 from the Institute of Space Studies of Catalonia, Spain; and the Ph.D. degree in Physics in 2002, from the University of Barcelona, Spain.

He is currently with the Unidad de Tecnología Marina (UTM - CSIC), Barcelona, Spain, working on satellite remote sensing. In particular, he is involved in scatterometry and L-band radiometry.

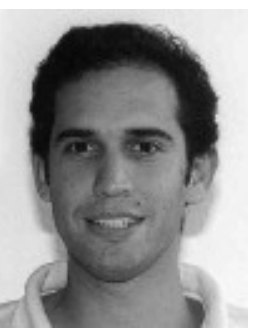

